\title{
Retour du congrès EuroMedLab des représentants de la SFBC
}

\section{Barcelone, 19-23 mai 2019}

\section{Bruno Baudin \\ Guilaine Boursier \\ Vincent Delatour \\ Philippe Gillery \\ Bernard Gouget \\ Damien Gruson \\ Laurence Piéroni \\ Michel Vaubourdolle}

Représentants de la Société française

de biologie clinique (SFBC)

$<$-pieroni@chu-montpellier.fr>

\section{Euromedlab, un événement important de veille scientifique en biologie clinique}

Bernard Gouget $^{1}$, Damien Gruson ${ }^{2}$, Sergio Bernardini ${ }^{2}$

${ }^{1}$ Comité des relations internationales de la SFBC, Président du Committee on mobile health and bioengineering in laboratory medicine (C-MHBLM) ; ${ }^{2}$ International federation of clinical chemistry and laboratory medicine executive committee, division on emerging technologies (EC-ETD)

$<$ b.gouget@icloud.com>

Maurizio Ferrari, past-IFCC président, a assuré la présidence du comité d'organisation (COC). Damien Gruson, (comité exécutif de la division IFCC sur les technologies émergentes (IFCC-ETD)) et Michel Vaubourdolle, (SFBC et l'EFLM, comité international (ISAB)) représentaient respectivement la Société royale belge de médecine de laboratoire (SRBML) au CS et la SFBC au Comité international consultatif (ISAB). Organisé tous les deux ans, cet évènement international a permis non seulement de mettre à jour ses connaissances, mais aussi d'avoir l'opportunité de découvrir les derniers développements technologiques et des recherches en biologie clinique et médecine de laboratoire afin d'anticiper les mutations à venir.

Le programme, réparti sur cinq jours, s'articulait autour de 20 symposia, 40 ateliers, plusieurs débats, 7 sessions thématiques et quatre conférences plénières. 1563 communications affichées provenant de 83 pays ont fait éclater les précédents records, la présence des pays hispaniques y étant pour beaucoup.
La conférence inaugurale présentée par Dr Manuel Serrano, Institut de recherche en biomédecine, Barcelone, Espagne, a abordé les progrès réalisés dans la compréhension des mécanismes du vieillissement et des cellules sénescentes et les applications médicales potentielles. Parmi les sujets traités au cours du congrès, on peut citer : l'épigénétique appliquée au cancer, le microbiome, les big data et la sécurité des données, les outils moléculaires CRISPR, le diabète, les maladies rénales, hépatiques et de la thyroïde, la septicémie, la fécondation in vitro, les maladies cérébro-vasculaires et cardiaques, l'immunosuppression personnalisée, les erreurs métaboliques congénitales, les tests prénatals non invasifs de l'ADN circulant, la biologie délocalisée et les dyslipidémies. Plusieurs sessions furent consacrées au management de la qualité et à l'évolution de la profession notamment dans un contexte européen. La présence de «IFCC-young scientists » enthousiastes a été appréciée.

\section{Réunion des National society journal editors and publishers}

\section{Laurence Piéroni}

Rédactrice en chef des Annales de Biologie Clinique

<l-pieroni@chu-montpellier.fr>

Cette réunion s'est tenue le lundi 20 mai et a permis aux éditeurs des journaux des sociétés savantes de confronter leurs problématiques, qui, quelle que soit la notoriété du journal dont ils ont la charge, sont identiques. 
La première (présentée par Nader Rifai, Clinical Chemistry) réside dans la difficulté de trouver des reviewers, compétents et disponibles pour assurer la revue d'articles dans des délais raisonnables, afin de permettre aux auteurs de publier leurs travaux sans avoir à retravailler leurs références bibliographiques.

Les éditeurs comparent leur taux de rejet immédiat des articles qu'ils reçoivent et s'accordent sur un taux d'environ $50 \%$ (voire plus pour Clin Chem), ce qui constitue une lourde charge pour les éditeurs en chef et leur équipe d'éditeurs associés.

Des idées sont ensuite soumises pour améliorer la qualité des articles, car de nombreux articles, d'après les informations que font remonter les reviewers aux éditeurs, sont soumis par ordre décroissant d'impact factor à Clin Chem, Clin Biochem, CCLM, etc. Les problèmes concernant le respect des topics des journaux ainsi que ceux liés aux analyses statistiques pourraient être résolus si les recommandations aux auteurs étaient respectées. Une proposition est approuvée par l'ensemble et des séances de travail pourraient être proposées sur le processus de review aux sessions des « young scientists».

Un problème et non des moindres est soulevé par l'éditrice de Biochemica, qui a fait face à la violation des droits d'auteurs par des journaux, qui ont édité sur internet des volumes complets du journal.

Enfin, Joris Delanghe a parlé de la soumission simultanée par des auteurs qui ne souhaitent pas attendre la fin d'un processus de revue avant de proposer leur travail à un autre journal. Là encore, les éditeurs qui ont rencontré ce problème proposent d'informer les auteurs qu'ils seront persona non grata dans leur journal.

En conclusion, chers lecteurs et auteurs, n'hésitez pas à devenir reviewers !

\section{Réunion du comité IFCC point of care testing (C-POCT)}

\author{
Michel Vaubourdolle \\ Comité des relations internationales de la SFBC, Full mem- \\ ber IFCC C-POCT \\ $<$ michel.vaubourdolle@aphp.fr>
}

Le comité C-POCT fait partie de la division « éducation et management » de l'IFCC et regroupe une dizaine de membres autour d'une réflexion sur l'utilisation, l'optimisation et l'harmonisation des pratiques en matière de biologie délocalisée au sens large (« examens réalisés à proximité du patient ou à l'endroit où il se trouve, dont le résultat peut entraîner une éventuelle modification des soins prodigués au patient »). Des informations peuvent être trou- vées sur http://www.ifcc.org/ifcc-education-division/emdcommittees/taskforcepoct/.

Le chairman, Adil Khan, accueille le 21 mai 2019 à $13 \mathrm{~h} 30$ 12 membres du groupe dans la salle et 2 en conférence téléphonique.

Le premier point concerne l'organisation du symposium satellite POCT qui se tiendra à Séoul, Corée, le 24 mai 2020 en marge du WorldLab2020. Un programme est élaboré en séance pour faire intervenir les membres du groupe et des spécialistes coréens. Parmi les thèmes qui seront abordés, nous pouvons citer : glucomètres, situation du POCT en Corée, rôle du LBM dans le management du réseau POCT, aspects récents des dispositifs délocalisés utilisant les techniques de biologie moléculaire, rôle du POCT en réanimation pour une goal-directed therapy, intérêt médicoéconomique de la thromboélastographie délocalisée dans la gestion optimisée des produits sanguins labiles...

En deuxième point, les résultats du sous-groupe travaillant sur l'évaluation des glucomètres chez les patients critiques seront résumés dans un document avant la fin de l'année.

En troisième point, le groupe travaille sur un article (position paper) pour publier des recommandations sur le management de la qualité des examens délocalisés dans différentes situations au sein de l'hôpital et en dehors (cabinets médicaux, TROD, autotests). La notion de risque patient est essentielle pour proposer dans chaque situation un niveau adapté d'assurance de la qualité.

Enfin, Sverre Sandberg, représentant l'EFLM, propose au groupe de construire un atelier POCT lors de l'EuroMedLab 2021 à Munich. Cette proposition sera étudiée prochainement.

\section{Session «De nouveaux développements pour d'anciennes maladies »}

Bruno Baudin

Secrétaire général adjoint SFBC

$<$ bruno.baudin@aphp.fr>

Cette session était dédiée aux marqueurs des maladies chroniques, tant les anciens que ceux en développement. Trois grands thèmes ont été traités : les marqueurs cardiaques (P. Laitinen, Finlande), le diabète (S. Atkin, Qatar) et l'insuffisance rénale chronique (E. Cavalier, Belgique). La conférence devant être donnée par H. Morris sur la vitamine $\mathrm{D}$ a été annulée, l'orateur étant décédé quelques jours auparavant ; un hommage lui a été rendu.

$\mathrm{Au}$ sujet des marqueurs cardiaques, P. Laitinen a insisté sur le besoin de standardisation des troponines et des BNP/NT-proBNP. On s'intéresse maintenant aux complexes TnI/TnT. Les marqueurs d'insuffisance cardiaque sont 
en plein développement, car à côté des classiques BNP et troponines, on trouve, entre autres, l'ANP, la MR-proADM, la copeptine, la galectine 3, le GDF-15 et des miRNA.

L'intervention de S. Atkin sur le diabète a surtout concerné les nouveaux traitements, la biologie n'apparaissant qu'en élément de contrôle des effets indésirables comme l'acidose métabolique (mesures du $\mathrm{pH}$ et du 3-hydroxybutyrate), l'insuffisance rénale (dosage de créatinine) et le déficit en vitamine B12 (la mesurer).

E. Cavalier a donné une conférence historico-scientifique sur la créatinine, de sa découverte à la standardisation par ICP-MS, en passant par les méthodes colorimétriques ou enzymatiques, et bien sûr les formules pour déterminer le débit de filtration glomérulaire (DFG) jusqu'à la formule CKD-EPI. On sait maintenant que la cystatine C n'apporte pas de valeur ajoutée à la créatinine, et que l'injection d'iohexol, dosé par LC-MS/MS dans le sang, est la meilleure méthode pour mesurer un DFG vrai (un seul laboratoire par pays devrait suffire). La métabolomique propose des panels de biomarqueurs d'insuffisance rénale ; par UPLC-MS/MS, ont été successivement proposés un panel à 15 biomarqueurs (dans lesquels on trouve des acides aminés, des oses et la créatinine !) et un panel à seulement 4 biomarqueurs (acides aminés et bases puriques, sans la créatinine !) ; fin 2019, leurs performances devraient être comparées à celles de la créatinine seule.

\section{Symposium «Questions éthiques en biologie médicale »}

\section{Guilaine Boursier}

Full member Task force young scientists and EFLM WG Accreditation and ISO/CEN standards

<g-boursier@chu-montpellier.fr>

La conférence «questions éthiques en biologie médicale » était une conférence d'actualité pour les biologistes français, notamment en raison de la révision de la loi de bioéthique actuellement en discussion à l'Assemblée. Matthew McQueen (CA) a tout d'abord rappelé la définition de l'éthique, un mot provenant du grec ethikos et décrivant les caractères et les croyances d'une communauté, d'un système ou d'une personne. Il a postulé qu'en tant que professionnels santé impliqués dans les laboratoires, nous devions définir une éthique professionnelle. La déontologie que nous connaissons en France ne semble pas être aussi clairement encadrée dans tous les pays du monde. Matthew McQueen et Maurice O'Kane (GB) ont ainsi rappelé les grandes lignes de l'éthique en biologie médicale : confidentialité des données médicales, allocation juste des ressources, la déclaration des conflits d'intérêts, la publi- cation scientifique de données vérifiées qui peuvent être reproduites.

Selon Jon Johannes Jonsson (IS), l'éthique nous aide à faire des choix et à faire face à l'incertitude. Ce thème est d'autant plus important en médecine génomique. Jon Johannes Jonsson a partagé sa pratique en tant que généticien sur la communication des découvertes secondaires aux patients. Les découvertes secondaires, en génétique, sont les variants potentiellement pathogènes ou clairement pathogènes, qu'on retrouve dans des gènes qui ne sont pas les gènes cibles de la maladie explorée. Ces découvertes sont de plus en plus fréquentes avec l'utilisation du séquençage de nouvelle génération (NGS) en routine dans nos laboratoires, et notamment des séquençages pangénomiques (exome et génome). Une liste de 59 gènes actionnables a été définie par la Société américaine de génétique. L'identification d'un variant pathogène dans un de ces gènes implique une modification de la prise en charge ou un nouveau diagnostic fortuit. Les moyens de communication de ces découvertes secondaires en France ne font pas encore l'unanimité dans la profession. Néanmoins, la Société française de médecine prédictive et personnalisée a récemment publié des recommandations de prise en charge des patients concernés. Jon Johannes Jonsson a proposé cette communication à 47000 patients pris en charge dans son laboratoire. Parmi ces patients, 370 étaient porteurs d'un variant pathogène de BRCA2, 40 en avait déjà connaissance et 220 ont été recontactés pour une consultation de génétique. Les autres patients n'ont pu être recontactés. De plus, 100 apparentés ont appelé le service après qu'un membre de leur famille ait reçu l'information d'une découverte secondaire. Les conseils génétiques pouvaient être proposés lors d'une consultation ou par téléphone : la majorité des patients ont choisi le téléphone. Les patients étaient ensuite revus en consultation pour leur suivi si nécessaire. Son retour était très positif et le parcours de soins du patient a été bien défini au sein de sa structure. Il existe d'autres situations où le médecin est initialement exclu du parcours de soins, il s'agit du cas des autotests, ces tests vendus directement aux patients sans l'intermédiaire d'un personnel de santé. Anne Cambon-Thomsen (FR) est impliquée dans un groupe de travail sur ces sujets de sociétés et notamment dans le domaine de la génétique. Ces tests sont actuellement interdits en France et il est illégal pour un citoyen français d'acheter ce type de tests génétiques, même sur internet. Néanmoins, le marché des autotests de génétique aux États-Unis atteindra jusqu'à 2,5 milliards de dollars en 2024 avec un taux de croissance de $12 \%$ (source : Directto-consumer genetic testing market). Parmi les autotests de génétique liés à la santé, plus de $50 \%$ sont des tests permettant de s'informer sur son risque cardiovasculaire. Plusieurs études se sont intéressées à l'influence de ces résultats sur les patients acheteurs de ces tests et aucune n'a mis en 
évidence d'effets franchement négatifs ou franchement positifs de ces résultats sur le comportement des patients vis-à-vis de leur santé. Aucune incompréhension ne semble être générée par la communication de ces résultats d'autotest. Selon Kaufman et al., $94 \%$ des autotests de génétique seraient réalisés à des fins de curiosité médicale et $90 \%$ pour connaître son statut vis-à-vis d'une maladie héréditaire ou ses origines généalogiques éloignées. Plus de la moitié des personnes interrogées ont déclaré avoir appris une information qui peut les aider à améliorer leur été de santé. Bien que les patients ont « le droit de savoir », l'oratrice a insisté sur les questions éthiques autour de ces autotests : l'information et le recueil du consentement inexistant ou insuffisant, l'absence de protection des individus face à aux résultats, la régulation très laxiste concernant les patients mineurs, la confusion possible entre la « recherche » et le soin, l'insuffisante communication sur la commercialisation des données de génétiques recueillies, l'absence de circuit médical accompagnant les patients qui le nécessiterait et le recours systématique aux analyses de génétique. Selon Anne Cambon-Thomsen, ces autotests font partie intégrante des tests génétiques mais une régulation, une meilleure information aux patients et une assurance sur la qualité de ces autotests sont nécessaires.

\section{Focus sur les technologies émergentes}

\author{
Bernard Gouget ${ }^{1}$, Damien Gruson ${ }^{2}$, Sergio Bernardini ${ }^{2}$ \\ ${ }^{1}$ Comité des relations internationales de la SFBC, Président \\ $\mathrm{du}$ Committee on mobile health and bioengineering in labo- \\ ratory medicine (C-MHBLM) ; ${ }^{2}$ International federation \\ of clinical chemistry and laboratory medicine executive \\ committee, division on emerging technologies (EC-ETD)
}

$<$ b.gouget@icloud.com>

La division IFCC sur les technologies émergentes (IFCCETD) a réuni son comité exécutif et ses trois comités : Comité sur les technologies emergentes en pédiatrie (CETPLM, Chair T. Lang), Comité sur santé mobile et bio-ingéniérie en médecine de laboratoire (C-MHBLM, Chair B. Gouget (FR) et Comité sur les omics (C-OT, Chair : G.J. Tsongalis). L'ensemble des membres avaient pour mission de repérer les dernières innovations technologiques, les nouveaux outils de l'automation et de la robotique et les applications du numérique proposées par les grands groupes IVD. Sergio Bernardini, chair ETD a par ailleurs coordonné la session sur les «Big data en biologie médicale ».

Premier orateur, D. Gruson, Université de Louvain, a souligné le fort potentiel des données de santé pour le développement de la médecine de précision et les applications de l'intelligence artificielle en santé. L'exploitation des données présente de nombreux intérêts : identification de facteurs de risques, aide au diagnostic, suivi thérapeutique, et en épidémiologie. Il a souligné les succès en analyse d'images, en radiologie, en pathologie, en dermatologie et en analyse génomique. Les big data n'en soulèvent pas moins des nombreux défis techniques et des questions éthiques.

L'intelligence artificielle (IA) est un domaine de recherche au cœur de la médecine du futur. La robotique en pleine expansion, sous-domaine spécifique de l'IA, vise à augmenter l'autonomie des machines en les dotant de capacités perceptuelles, décisionnelles et d'action. L'IA a clairement le potentiel de générer une nouvelle révolution et un changement de paradigme en médecine de laboratoire avec un suivi des patients à distance, des diagnostics personnalisés grâce au regroupement d'un nombre croissant de données. Son potentiel augmente de façon exponentielle en raison du flux, de la quantité et de la variété des données disponibles qui constituent des bases de données à exploiter. L'ADN et de nombreuses modifications épigénétiques, l'ARN sous diverses formes : messager, à double brin non codant ou double brin, les protéines, le métabolome ou le microbiote peuvent être ainsi mesurés, et les données cliniques, médicales et environnementales mieux structurées. L'accessibilité aux données reste encore très complexe en raison du manque de standardisation, d'utilisation de différents logiciels, de différences de syntaxe et de terminologie. Le déploiement de l'IA suppose des investissements financiers et un cadre réglementaire et financier, national et international adapté.

Larry Kricka, Department of pathology and laboratory medicine, University of Pennsylvania medical center, Philadelphia, PA, USA nous a offert un brillant panoramique des technologies émergentes. La médecine de laboratoire est un domaine unique en soi. Des techniques d'analyse de données capables d'analyser des ensembles de données volumineux et complexes ont été récemment développées. Ces méthodes analytiques à haute résolution qui génèrent des téraoctets d'informations, sont désormais monnaie courante (cf, le séquençage de l'ADN). De nouvelles technologies génératrices de données volumineuses d'une complexité différente continuent à émerger, par exemple une méthode métabolomique par résonance magnétique nucléaire pour l'analyse du sang qui teste 220 biomarqueurs, et l'analyse de l'haleine (volatolomics) à l'aide de réseaux de capteurs à base de nanomatériaux se développe. La surveillance continue est aussi un moyen d'acquérir de grandes quantités de données. De nombreux « dispositifs portables », appartenant à la révolution de la santé mobile (mHealth), permettent de suivre un éventail d'indicateurs de santé (par exemple, l'électrocardiogramme, la fréquence cardiaque, l'activité et paramètres biologiques). L'Apple watch et the Virly study watch sont de bons exemples. Ces 
dispositifs pratiques et conviviaux sont utilisés pour collecter des données dans de grandes études de population comme l'étude sur 10000 patients atteints de la maladie de Parkinson, et la caméra sur smartphones a été utilisée dans une gamme de tests cliniques comprenant l'analyse d'urine, la détection de calcul rénaux, ou de cancer de la peau. L'intelligence artificielle (IA) et la réalité augmentée sont au premier plan pour faciliter l'analyse des images médicales. Les algorithmes pour l'apprentissage en profondeur «Robust AI » (deep learning), développés pour analyser des images de tomographie de cohérence optique 3D, peuvent égaler voire dépasser la précision du raisonnement humain. Un microscope, doté d'un système à réalité augmentée, utilise des algorithmes de machine «learning » pour analyser des images de microscope puis sélectionner et projeter des zones à examiner dans le champ de vision, agissant ainsi comme un assistant intelligent. Parmi les autres applications de l'IA figurent l'analyse des données génétiques, la télé-assistance et les jumeaux numériques (avatars). Un aspect important concerne la cybersécurité des dispositifs médicaux et l'importance croissante des GAFAM qui s'intéressent aux outils de collecte, de stockage, d'analyse de données médicales, et de la santé mobile.

En médecine de laboratoire, les big data sont largement générés pour la recherche génomique et la médecine de précision. L'IA et le big data sont plein de promesses pour les entreprises de toutes les industries, y compris en santé. Toutefois, le véritable potentiel révolutionnaire de ces deux technologies repose probablement sur leur convergence. La recherche en génomique s'intéresse au diagnostic et à la thérapeutique de maladies spécifiques en utilisant les technologies de séquençage de nouvelle génération, telles que le séquençage du génome entier, le séquençage de l'exome complet, le séquençage de l'ARN et/ou le séquençage ciblé de gènes. Les informations obtenues à partir de données NGS à grande échelle sont combinées de manière exhaustive avec les données de dossiers médicaux électroniques pour aborder la maladie en vue d'un traitement et d'une médecine spécialisée.

Progresser sur la connaissance de ces pathologies nécessite la mise en œuvre de méthodes d'analyse capables de gérer des volumes importants de données et une infrastructure informatique (logiciels, algorithmes) puissante. Ces données multidimensionnelles à grande échelle peuvent être analysées directement pour des caractéristiques telles que des mutations ou des processus pathogènes, et/ou des analyses complexes, y compris des approches intégrées d'algorithmes d'apprentissage automatique de prédiction et de stratification de l'intelligence artificielle. Les «clouds » d'Amazon Web Services (AWS) sont un exemple de niche de service fournissant la vitesse d'analyse, la sécurité, le stockage, et les infrastructures appropriées telles que la base de données APACHE Hadoop et NOSQL. Globalement, ces méga-données générées offrent un large éventail d'informations stratégiques sur la prévention des maladies, le traitement et les diagnostics cliniques.

La question est de savoir comment utiliser efficacement les données de santé dans la révolution qu'apportent l'intelligence artificielle (IA) et le machine learning dans le domaine de la santé. L'IA est en plein essor, de nombreuses voies de recherche sont explorées pour améliorer les performances techniques de ces systèmes, mais aussi leur adéquation aux pratiques médicales. Les voies de recherche portent en particulier sur le traitement des données et leur qualité, mais aussi sur la conception de systèmes transparents pour l'utilisateur et bien adaptés au contexte d'utilisation.

Les innovations modernes exigent souvent des concepts issus de plusieurs domaines d'expertise. On ne peut que vivement recommander aux jeunes scientifiques de participer à ces évènements internationaux, pour acquérir une formation, approfondir, échanger. Bénéficier d'une expérience à l'international est toujours enrichissant. La SFBC à un programme de bourses ambitieux. À vous de jouer !

\section{Session « Recommandations relatives aux dyslipidémies et nécessité de standardiser les biomarqueurs traditionnels et les nouveaux marqueurs des maladies cardiovasculaires »}

\section{Vincent Delatour $^{1}$, Huu Hien Huynh ${ }^{2}$, Philippe Gillery ${ }^{3}$}

${ }^{1}$ Membre du Groupe de travail IFCC sur les apolipoprotéines (IFCC WG-ApoMS) ; ${ }^{2}$ Laboratoire national de métrologie et d'essai (LNE) et ESPCI Paris, Spectrométrie de masse biologique et protéomique, CNRS USR 3149, Paris, France ; ${ }^{3}$ Chargé des relations internationales de la SFBC, Président de la division scientifique de l'IFCC

$<$ vincent.delatour@lne.fr>

Les modérateurs de la session étaient Philippe Gillery (Reims, France) et Christa Cobbaert (Leiden, Pays Bas). La présentation de Philippe Gillery s'est attachée à montrer l'importance d'assurer la fiabilité des examens de biologie médicale pour permettre un diagnostic et un suivi adaptés des patients. Le raccordement métrologique des résultats aux étalons internationaux et aux méthodes de référence constitue un moyen privilégié pour assurer la comparabilité et la justesse des dosages, répondant ainsi à des exigences clés de la norme ISO 15189, de la directive 98/79/CE et du règlement 2017/746 relatifs aux dispositifs médicaux de diagnostic in vitro (DM DIV). Pour qu'elles soient 
couronnées de succès, il est nécessaire que les actions de standardisation impliquent dès le début les différentes parties prenantes : biologistes, cliniciens, autorités de santé, laboratoires de métrologie et fabricants de trousses de diagnostic in vitro.

La présentation de Michel Langlois (Gand, Belgique) était focalisée sur les recommandations de l'EFLM (European federation of clinical chemistry and laboratory medicine) relatives au diagnostic et au suivi des dyslipidémies, et en particulier l'analyse des lipoprotéines athérogènes dans un contexte de développement de la médecine personnalisée. Actuellement, les dosages simultanés du cholestérol total (CT), des triglycérides (TG), du cholestérol HDL (cHDL) et du cholestérol-LDL (cLDL) (ou son calcul) sont les plus utilisés, mais ils présentent des limitations importantes, suggérant que d'autres paramètres seraient plus adaptés. Tout d'abord, les performances analytiques des dosages directs de cLDL apparaissent insuffisantes aux très faibles concentrations de cLDL et/ou lorsque la concentration de TG est élevée. Ensuite, il apparaît qu'avec un suivi basé sur la détermination du cLDL, le risque résiduel est très élevé : des risques résiduels compris entre 60 et $76 \%$ dans 8 essais cliniques majeurs sont évoqués. Le choix de tests de diagnostic appropriés apparaît particulièrement important dans des situations où de fortes réductions de la concentration de cLDL, qui peuvent être obtenues avec des traitements combinés (e.g. statines + inhibiteurs de PCSK9), sont recommandées. Différentes questions ont été discutées :

- quelles sont les lipoprotéines athérogènes présentant un intérêt ? Remannts, LDL et Lp(a) apparaissent comme les cibles prioritaires ;

- quels sont les meilleurs biomarqueurs pour estimer le risque cardiovasculaire? De par les limitations des dosages de cLDL aux faibles concentrations de cLDL et/ou aux concentrations élevées de TG, le dosage de l'ApoB ou du cholestérol non-HDL (non-cHDL) apparaissent préférables. La non prise en compte du cholestérol associé à la $\mathrm{Lp}(\mathrm{a})$ et aux remnants, le biais des dosages de cHDL dans les échantillons dyslipidémiques, ou la surestimation du cVLDL dans des échantillons hypertriglycéridémies seraient susceptibles d'expliquer la non-réponse ou le manque de réponse aux traitements ;

- est-il temps de remplacer le dosage du cLDL par des analyses avancées de lipoprotéines ? Tandis que le noncHDL apparaît être l'option la moins coûteuse et le dosage de l'ApoB une alternative satisfaisante, le profilage d'apolipoproteines par LC/MS/MS et la détermination de la concentration en nombre et de la taille de l'ensemble des sous-classes de lipoprotéines par RMN ou mobilité ionique apparaissent être des technologies d'avenir, mais qui restent peu utilisées car onéreuses.
La présentation de Vincent Delatour (Paris, France) s'est attachée à montrer la difficulté de standardiser les analyses avancées de lipoprotéines et notamment les mesures de concentration en nombre de classes et sous-classes de lipoprotéines. Dans le cadre du projet européen de métrologie BioSITrace, une inter-comparaison des différentes techniques de dosage du non-HDL-P a été organisée. Les résultats montrent une variabilité inter-technique de $14 \%$, ce qui est à la fois trop élevé (les différentes techniques ne fournissent pas des résultats équivalents) et paradoxalement une bonne surprise compte tenu du fait que les différentes méthodes reposent sur des principes physiques très différents et ne mesurent sans doute pas la même chose. La LC/MS/MS apparaît comme la méthode la plus à même de devenir la méthode de référence avec laquelle toutes les autres seraient standardisées : d'une part, car elle permet de raccorder les résultats au système international d'unités et, d'autre part, car sa sélectivité permet de mesurer des entités bien définies et enfin car les incertitudes de mesure sont suffisamment faibles. Tandis que les dosages d'ApoB/non-HDL-P pourront être standardisés par LC/MS/MS (un groupe de travail de l'IFCC (International federation of clinical chemistry and laboratory medicine) sur la standardisation des dosages d'apolipoprotéines par spectrométrie de masse a été créé à cette fin), cela n'est pas le cas de l'HDL-P ni des autres sous classes de lipoprotéines. Il apparaît que la standardisation des analyses avancées de lipoprotéines sera difficile et prendra du temps. Le projet EMPIR CardioMet s'attachera à : 1) documenter les performances des dosages actuellement réalisés et établir des recommandations sur les performances analytiques que peuvent et doivent atteindre les méthodes de routine, 2) soutenir les travaux du groupe de travail de l'IFCC à travers le développement d'étalons internationaux et de méthodes de référence pour le dosage des apolipoprotéines, 3) évaluer la valeur ajoutée des analyses avancées de lipoprotéines pour réduire le risque résiduel non diagnostiqué.

La présentation de Christa Cobbaert (Leiden, Pays Bas) a montré l'état d'avancement des travaux du groupe de travail de l'IFCC sur la standardisation des dosages d'apolipoprotéines par spectrométrie de masse. L'objectif de ces travaux consiste à développer une méthode de référence primaire pour le dosage (si possible simultané) des apolipoprotéines A-I, B100, B48, C-I, C-II, C-III, C-IV, E et de la Lp(a) par LC/MS/MS. Les 2 principaux challenges sont d'une part, de démontrer que la digestion enzymatique est complète et, d'autre part, de disposer d'étalons primaires (protéines recombinantes ou peptides synthétiques) dont la pureté a été rigoureusement évaluée. La mise au point d'une méthode multiplex implique de développer des techniques de préparation d'échantillon optimales pour les différentes apolipoprotéines (par exemple durée de la 
digestion enzymatique), ce qui nécessite certains compromis. À l'avenir, il est probable que le profilage d'apolipoprotéines par LC/MS/MS ne sera pas utilisé exclusivement à des fins de standardisation mais également dans le cadre du suivi de patients à haut risque cardiovasculaire pour lesquels il existe un important risque résiduel non diagnostiqué par les techniques conventionnelles. D'une manière plus générale, les approches protéomiques reposant sur l'analyse quantitative de panels de biomarqueurs protéiques par spectrométrie de masse apparaissent comme très prometteuses pour une médecine plus personnalisée. 